\title{
High genetic diversity in hard ticks from a China-Myanmar border county
}

\author{
Lan-Hua Li ${ }^{1}$, Yi Zhang ${ }^{2}$, Jia-Zhi Wang ${ }^{3}$, Xi-Shang Li ${ }^{3}$, Shou-Qin Yin ${ }^{3}$, Dan Zhu², Jing-Bo Xue ${ }^{2}$ and Sheng-Guo Li ${ }^{3 *}$
}

\begin{abstract}
Background: Many tick species have great morphological similarity and are thus grouped into species complexes. Molecular methods are therefore useful in the classification and identification of ticks. However, little is known about the genetic diversity of hard ticks in China, especially at the subspecies level. Tengchong is one of the epidemic foci of tick-borne diseases in China, but the tick species inhabiting the local area are still unknown.

Methods: Eighteen villages in Tengchong County, China, were selected for sampling carried out from September to October 2014. Infesting hard ticks were removed from the body surface of domestic animals and questing ticks were collected from grazing fields. After morphological identification, molecular characteristics of each tick species were analyzed based on both 165 rRNA and cytochrome $c$ oxidase subunit 1 (cox 1 ) gene fragments.
\end{abstract}

Results: Six tick species were identified based on morphology: Rhipicephalus microplus, R. haemaphysaloides, Ixodes ovatus, Haemaphysalis longicornis, H. shimoga and H. kitaokai. Phylogenetic analysis using the cox 1 gene revealed that $R$. microplus ticks from the present study belong to clade C. For tick samples of both $R$. haemaphysaloides and I. ovatus, three phylogenetic groups were recognized, and the intergroup genetic distances exceeded the usual tick species boundaries. Haemaphysalis longicornis ticks were clustered into two separate clades based on the cox1 gene. For ticks from both H. shimoga and H. kitaokai, two phylogenetic groups were recognized based on the phylogenetic analysis of the 16S rRNA gene, and the intergroup genetic distances also exceeded the known boundaries for closely related tick species.

Conclusions: According to molecular analyses, new species or subspecies closely related to $R$. haemaphysaloides, I. ovatus, H. shimoga and H. kitaokai probably exist in the China-Myanmar border Tengchong County, or these ticks form species complexes with highly divergent mitochondrial lineages. Morphological comparisons are warranted to further confirm the taxonomic status of these tick species.

Keywords: Hard tick, Genetic diversity, 165 rRNA gene, cox1 gene, Tengchong County, China

\section{Background}

Hard ticks (Acari: Ixodidae) are obligate blood-sucking parasitic arthropods which can infest reptiles, birds and mammals [1]. They transmit a wide range of pathogens of both veterinary and medical importance, including bacteria, viruses, protozoa, etc. Moreover, new pathogens are continuously being identified from ticks $[2,3]$. Therefore,

\footnotetext{
* Correspondence: tccdclsg@sina.com

${ }^{3}$ Tengchong Center for Disease Control and Prevention, Tengchong 679100, People's Republic of China

Full list of author information is available at the end of the article
}

hard ticks are usually considered to be the most important vectors of pathogens in the temperate zone [4].

China covers approximately 9.6 million square kilometers of land area. At least 117 tick species from seven genera have been reported in China [5]. In recent years, the reported number of tick-borne infections has increased in this country. Since 1982, more than 30 emerging tick-borne agents have been recognized [1, 6-9], including severe fever with thrombocytopenia syndrome virus, Borrelia burgdorferi (sensu lato), Babesia spp., the

(C) The Author(s). 2018 Open Access This article is distributed under the terms of the Creative Commons Attribution 4.0 International License (http://creativecommons.org/licenses/by/4.0/), which permits unrestricted use, distribution, and 
spotted fever group rickettsiae, Ehrlichia, Anaplasma, etc. Thus, tick-borne diseases are considered to be an emerging threat to public health in mainland China.

Identification of tick species is essential to the control of tick-borne diseases. However, traditional morphological identification requires extensive experience and can be challenging when the specimens are engorged with blood, in immature stages (larva or nymph stage) or physically damaged [10]. Therefore, molecular methods based on DNA barcoding, including the $16 S$ rRNA gene and the cytochrome $c$ oxidase subunit 1 ( $\operatorname{cox} 1)$ gene, are useful in the classification of ticks. Moreover, many tick species have great morphological similarity and are thus grouped into species complexes. Thus, molecular analysis can expand our knowledge of ticks. However, little is known about the genetic diversity of hard ticks in China, especially at the subspecies level.

Tengchong County of Yunnan Province is located on the southwest border of China $\left(24^{\circ} 38^{\prime}-25^{\circ} 52^{\prime} \mathrm{N}, 98^{\circ} 05^{\prime}-\right.$ $\left.98^{\circ} 45^{\prime} \mathrm{E}\right)$. It is adjacent to Myanmar on the west, and more than $70 \%$ of the land is covered by forest. The county is inhabited by a wide variety of wild animals and arthropods. Several human cases of tick-borne infections have also been reported there in recent years [11]. Hence, Tengchong is considered to be one of the epidemic foci of tick-borne diseases. However, the tick species inhabiting the local area are still unknown.

In the present study, we conducted a survey from September to October 2014, to investigate the distribution of tick species in Tengchong County. Molecular classification was then performed, and genetic diversity was analyzed for both the $16 \mathrm{~S}$ rRNA gene and the cox 1 mitochondrial gene.

\section{Methods}

\section{Tick collection and morphological identification}

A total of 18 villages with a relatively large amount of livestock were arbitrary selected from 14 towns of Tengchong County (Fig. 1 and Table 1). Infesting ticks were collected from the body surface of domestic animals, including cattle, goats and dogs, from September to October 2014. After collection of infesting ticks, questing ticks were collected with a white drag cloth

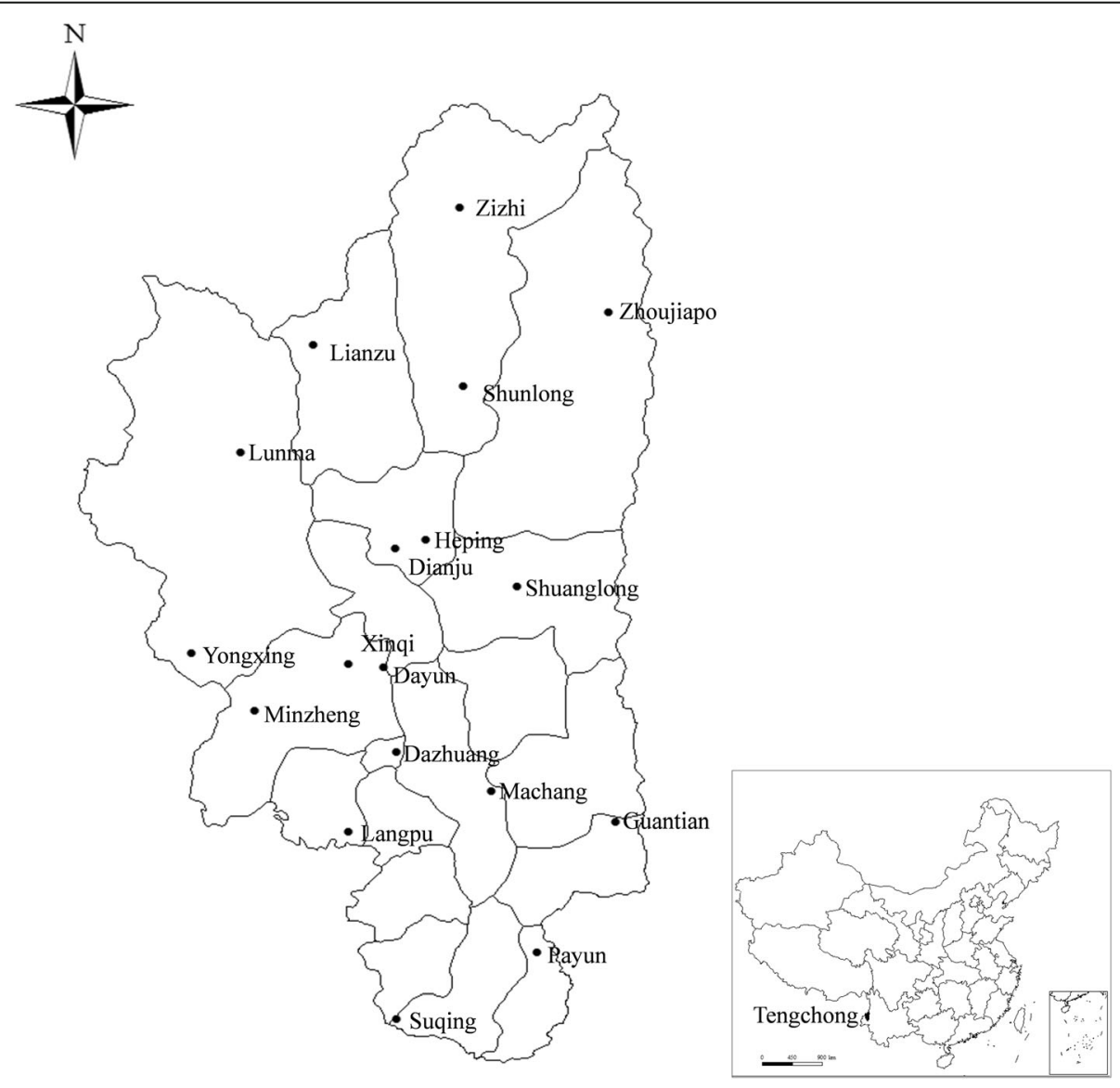

Fig. 1 Geographical locations for tick collection in the present study 
Table 1 Information on locations of tick collection and distribution of tick species in Tengchong County, Yunnan Province, China

\begin{tabular}{|c|c|c|c|c|c|c|c|c|c|}
\hline Location $^{a}$ & Latitude & Longitude & Elevation (m) & R. $m$. & R. h. & 1. 0. & H. I. & H. K. & H.S. \\
\hline Suqing, Xinhua & 24.68195 & 98.46520 & 1110 & $x$ & $x$ & & & & \\
\hline Langpu, Hehua & 24.92036 & 98.40881 & 1334 & & & & & & \\
\hline Payun, Tuantian & 24.76645 & 98.63097 & 1469 & & $\times$ & & & & \\
\hline Shuanglong, Qushi & 25.23325 & 98.60783 & 1542 & $x$ & & & & & \\
\hline Dazhuang, Heshun & 25.02243 & 98.46550 & 1579 & $x$ & $x$ & $\times$ & & & \\
\hline Minzheng, Zhonghe & 25.13423 & 98.40763 & 1724 & $\times$ & & $x$ & & & \\
\hline Dianju, Gudong & 25.29312 & 98.49935 & 1763 & $x$ & & & & & \\
\hline Yongxing, Houqiao & 25.14863 & 98.22210 & 1812 & $\times$ & $x$ & & & $\times$ & \\
\hline Heping, Gudong & 25.28138 & 98.46355 & 1812 & $\times$ & & & & & \\
\hline Xinqi, Zhonghe & 25.07495 & 98.29690 & 1824 & & & $x$ & & & \\
\hline Guantian, Wuhe & 24.93287 & 98.72402 & 1830 & $x$ & $x$ & $x$ & & & \\
\hline Shunlong, Mingguang & 25.48820 & 98.54453 & 1857 & $\times$ & & & $x$ & & \\
\hline Lunma, Houqiao & 25.40383 & 98.28007 & 1966 & $x$ & & & & $\times$ & $\times$ \\
\hline Zhoujiapo, Jietou & 25.58273 & 98.71622 & 1986 & $\times$ & & $x$ & & & \\
\hline Zizhi, Mingguang & 25.71568 & 98.54013 & 2059 & $x$ & & & $x$ & & \\
\hline Lianzu, Diantan & 25.54147 & 98.36693 & 2071 & $\times$ & & $x$ & & & \\
\hline Machang, Mangbang & 24.97200 & 98.57728 & 2163 & $x$ & & $x$ & $x$ & & \\
\hline Dayun, Mazhan & 25.12965 & 98.44957 & 2560 & $x$ & & $\times$ & & & \\
\hline
\end{tabular}

Abbreviations: R. m. Rhipicephalus microplus, R. h. R. haemaphysaloides, I. o. Ixodes ovatus, H. I. Haemaphysalis longicornis, H. k. H. kitaokai, H. s. H. shimoga ${ }^{a}$ Village, town

from the grazing locations. The ticks were preserved in $75 \%$ ethanol for further analysis. All ticks were identified to the species level under a dissecting microscope (Olympus Corporation, Tokyo, Japan) based on morphology [12]. The specimens were then stored at $-20{ }^{\circ} \mathrm{C}$ for subsequent processing.

\section{DNA extraction}

Ticks were rinsed 3 times for 1 min by vortex in $75 \%$ ethanol and then washed 3 times in sterile phosphate-buffered saline (PBS). DNA was extracted individually with a DNeasy Blood \& Tissue Kit (Qiagen, Hilden, Germany) according to the manufacturer's protocol.

\section{PCR amplification of the tick 165 rRNA gene and cox 1 gene}

For each tick species, at least 15 DNA specimens were selected for PCR amplification using primers targeting the $16 S$ rRNA gene fragment (16S+1: 5'-CCG GTC TGA ACT CAG ATC AAG T-3' and 16S-1: 5'-CTG CTC AAT GAT TTT TTA AAT TGC TGT GG-3') [13]. For tick species with less than 15 individuals, all of the DNA samples were PCR amplified. As two Ixodes ovatus groups were identified by subsequent molecular analysis, all of the specimens of I. ovatus ticks were PCR amplified using the primer set $16 \mathrm{~S}+1$ and $16 \mathrm{~S}-1$. The PCR was performed using a C1000 Touch $^{\text {тм }}$ Thermal Cycler (Bio-Rad Laboratories, Irvine, CA, USA) under the following conditions: $95^{\circ} \mathrm{C}$ for $5 \mathrm{~min}$, followed by 35 cycles of $95{ }^{\circ} \mathrm{C}$ for $30 \mathrm{~s}, 57{ }^{\circ} \mathrm{C}$ for $30 \mathrm{~s}$ and $72{ }^{\circ} \mathrm{C}$ for 40 s, ending at $72{ }^{\circ} \mathrm{C}$ for $10 \mathrm{~min}$. Each $20 \mu \mathrm{l}$ PCR mixture contained $10 \mu \mathrm{l}$ of $2 \times$ PCR Master Mix (with dyes, DBI ${ }^{\circ}$ Bioscience, Shanghai, China), $1 \mu \mathrm{l}$ of each primer (5 $\mathrm{mM}), 6 \mu \mathrm{l}$ of water and $2 \mu \mathrm{l}$ of DNA sample. The PCR products were then purified and sequenced by Sangon Biotech (Shanghai, China).

For each tick species, at least 2 specimens were selected based on the results of the molecular analysis of the $16 \mathrm{~S}$ rRNA gene. The selected specimens were further PCR amplified using primers targeting the cox1 gene (LC O1490: 5'-GGT CAA CAA ATC ATA AAG ATA TTG G-3' and HCO2198: 5'-TAA ACT TCA GGG TGA CCA AAA AAT CA-3') [14]. The PCR for the $\operatorname{cox} 1$ gene was performed under the same conditions as the amplification of the $16 S$ rRNA gene fragment.

\section{Molecular analysis of the tick $16 \mathrm{~S}$ rRNA gene and cox 1 gene}

Sequences of both the $16 S$ rRNA gene and the cox 1 gene were compared with the available data on GenBank using 
Blast on the NCBI website (https://blast.ncbi.nlm.nih.gov/ Blast.cgi) after trimming the low-quality sequences at both ends.

Representative $16 S$ rRNA or cox1 gene sequences of Rhipicephalus microplus, $R$. haemaphysaloides, $R$. sangui$n e u s$ and $R$. turanicus were downloaded from GenBank for phylogenetic analysis of Rhipicephalus species [15]. Similarly, $16 S$ rRNA or cox1 gene sequences of $I$. ovatus and species close to I. ovatus were downloaded from GenBank based on the results of BLAST, including I. vespertilionis and I. canisuga [16-18]. Using the same methods, representative sequences of several Haemaphysalis species were also downloaded, including $H$. shimoga, $H$. erinacei, $H$. cornigera, $H$. parva and $H$. kitaokai [19-23]. For $R$. microplus and $H$. longicornis, all of the $16 S \mathrm{rRNA}$ and cox 1 sequences of ticks from China were downloaded. Multiple sequence alignments were then conducted in Clustal W2, and the neighbor-joining (NJ) method was applied to construct a phylogenetic tree with 1000 replicates using MEGA 5.1 software [24].

To further determine the intraspecies and interspecies genetic distances among Ixodes, Rhipicephalus and Haemaphysalis ticks, pairwise Kimura's 2-parameter (K2P) distances were also calculated using the $16 S$ rRNA gene or $\operatorname{cox} 1$ gene in MEGA.

\section{Results}

\section{Morphological identification of ticks}

As shown in Table 2, 981 domestic animals were examined, including 837 goats, 113 cattle and 31 dogs. Among all the examined animals, 198 (20.2\%) were infested by ticks. A total of 903 infesting ticks were collected from the animals. Among all the infesting ticks, 652 were identified as $R$. microplus [25], 36 as $R$. haemaphysaloides [26], 29 as H. longicornis [26] and 186 as $I$. ovatus $[27,28]$ according to the identification key for adult ticks described by Deng \& Jiang [12]. Ixodes ovatus and $H$. longicornis were collected only from goats, $R$. microplus ticks were collected from both goats and cattle, and $R$. haemaphysaloides were found on all three animal species (Table 2).

A total of 558 questing ticks were collected. All of the questing ticks were larvae except for one $I$. ovatus adult. Among the questing larvae, 459 were identified as $R$. microplus (after Deng \& Jiang [12]; figure 352); 62 as $H$. longicornis (after Deng \& Jiang [12]; figure 167); 4 as $H$. shimoga [29]; and 32 as H. kitaokai [30].

\section{Molecular identification and classification of ticks by nucleotide BLAST}

Morphological identifications for $R$. microplus, $R$. haemaphysaloides and $H$. longicornis ticks were consistent with GenBank BLAST using sequences of both the $16 S$ rRNA and the cox 1 genes, with sequence identity of $98-100 \%$ to sequences of the corresponding tick species (Additional file 1: Table S1 and Additional file 2: Table S2).

For the $16 S$ rRNA gene sequences of $I$. ovatus from the present study, the closest sequence was from an $I$. ovatus isolate collected in Yunnan, China (GenBank: KU664519.1), with a sequence similarity of 92-97\% (Additional file 1: Table S1). A total of 15 specimens of I. ovatus were analyzed using the cox 1 gene. According to the results of the cox 1 gene BLAST, 2 I. ovatus specimens were closest to I. canisuga (GenBank: KY962 049.1 ), with a sequence similarity of $83 \%$, while 13 other I. ovatus ticks were closest to an I. ovatus isolate from Japan (GenBank: AB231670.1), with 87-88\% sequence similarity (Additional file 2: Table S2).

For the $16 S$ rRNA gene sequences of $H$. shimoga, the closest sequence was from a $H$. shimoga isolate collected from India (GenBank: MH044717.1), with a similarity of $95 \%$, while the sequences of $H$. kitaokai from this study were closest to the sequence of a $H$. kitaokai isolate from Japan (GenBank: AB819202.1), with a similarity of $94 \%$ (Additional file 1: Table S1). No cox 1 sequence of $H$. shimoga was available in the GenBank database; hence the cox 1 sequence closest to $H$. shimoga from this study was from I. erinacei (GenBank: KX901844.1), with a sequence similarity of $90 \%$. The closest cox 1 sequences to those for $H$. kitaokai generated here were from $H$. concinna (GenBank: JX573136.1), with a sequence similarity of $90 \%$ (Additional file 2: Table S2).

\section{Phylogenetic analyses and genetic distances for $R$. microplus and $\boldsymbol{R}$. haemaphysaloides}

At least 4 taxa have been reported in the $R$. microplus complex based on phylogenetic analysis of the $16 \mathrm{~S}$ rRNA

Table 2 Tick species infesting different domestic animals in Tengchong County, Yunnan Province, China

\begin{tabular}{|c|c|c|c|c|c|c|}
\hline \multirow[t]{2}{*}{ Animals } & \multirow[t]{2}{*}{$N$} & \multirow{2}{*}{$\begin{array}{l}\text { No. } \\
\text { infested } \\
(\%)\end{array}$} & \multicolumn{4}{|l|}{ Tick species } \\
\hline & & & R. microplus & R. haemaphysaloides & 1. ovatus & H. longicornis \\
\hline Goats & 837 & $149(17.8)$ & 258 & 2 & 186 & 29 \\
\hline Cattle & 113 & $47(41.6)$ & 394 & 18 & 0 & 0 \\
\hline Dogs & 31 & $2(6.5)$ & 0 & 16 & 0 & 0 \\
\hline Total & 981 & $198(20.2)$ & 652 & 36 & 186 & 29 \\
\hline
\end{tabular}




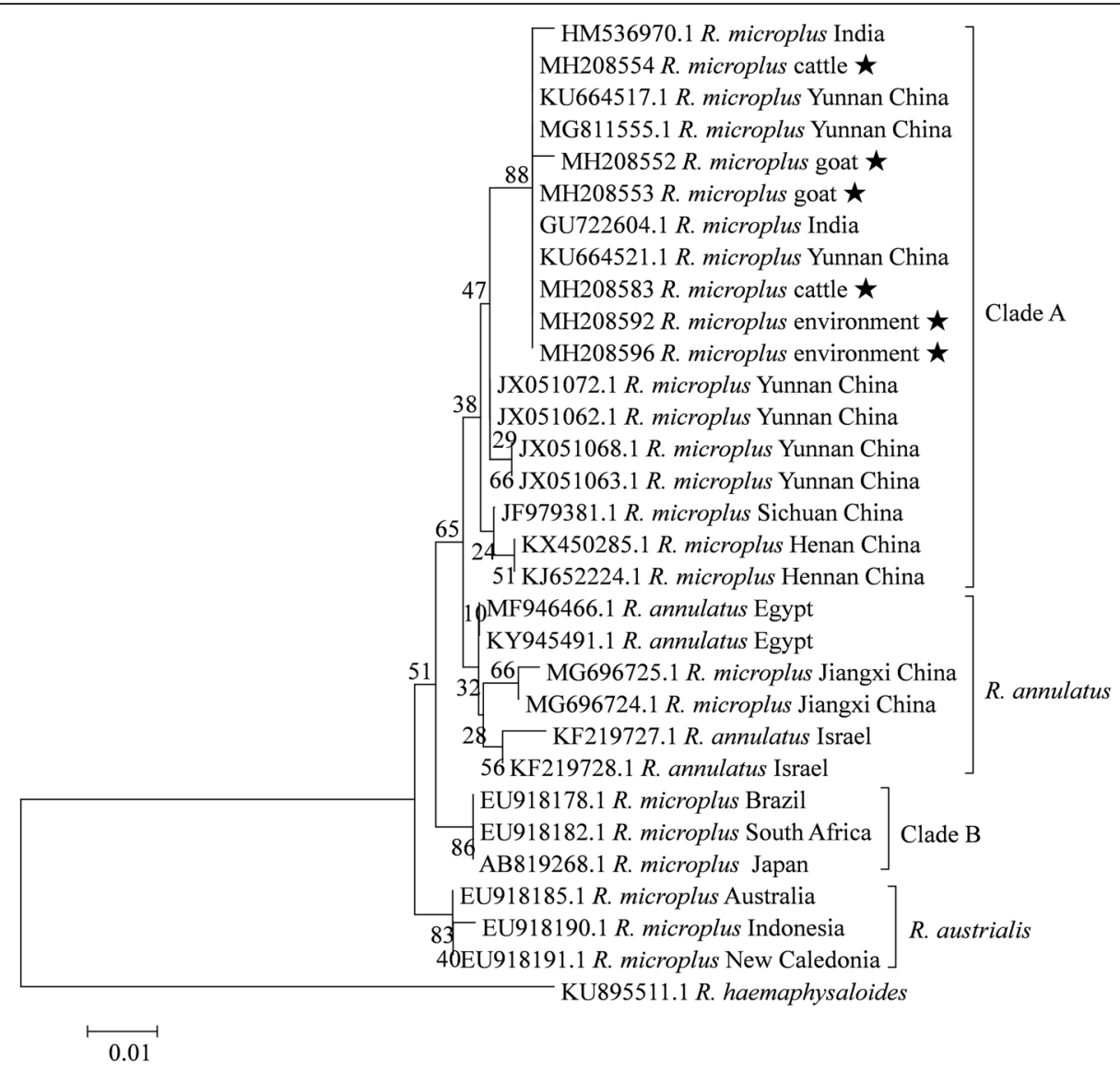

Fig. 2 Phylogenetic tree for R. microplus based on the 16S rRNA gene, including sequences obtained in the present study and representative sequences of the known subspecies-level taxa from GenBank. The sequences generated in the present study are indicated with a star

gene: $R$. annulatus, $R$. australis and $R$. microplus clades A and B [31, 32]. All R. microplus ticks collected during the present study were clustered into one clade, together with other $R$. microplus isolates from Yunnan Province of China and two Indian isolates (Fig. 2). The mean K2P distances within the group ranged between $0-0.008$, while the distances between groups ranged between 0.010-0.022 (Additional file 3: Table S3).

According to phylogenetic analysis based on the cox 1 gene, 5 taxa of $R$. microplus have been reported [31]. The ticks identified as $R$. microplus in the present study and two isolates from India clustered into clade B based on cox 1 gene analysis (Additional file 4: Figure S1). The mean K2P distances within and between groups ranged between $0-0.030$ and $0.059-0.100$, respectively (Additional file 5: Table S4).

For $R$. haemaphysaloides ticks, 36 sequences of $16 S$ rRNA gene fragments from the present study were analyzed, and 3 genetic groups were recognized. Twenty-six specimens from the present study and 10 from Taiwan of China formed Group 1; 2 ticks from India formed Group 2; and 12 specimens were clustered together and formed Group 3, including 10 from the present study, 1 from Thailand and 1 from Yunnan Province of China (Fig. 3). The mean K2P distances within groups ranged between $0-0.025$, while the mean distances between groups were $0.058-0.075$ (Additional file 6: Table S5).

\section{Phylogenetic analyses and genetic distances for Ixodes ovatus}

For I. ovatus ticks, 34 sequences of $16 \mathrm{~S}$ rRNA gene from the present study were selected for phylogenetic analysis, and 3 genetic groups of I. ovatus were recognized. Ixodes ovatus collected in Japan and the USA clustered together and formed Group 1, 7 specimens from the present study and one from another location of China formed Group 2, while other specimens from the present study formed Group 3 (Fig. 4). The mean K2P distances between groups ranged between 0.079-0.102, while the mean genetic distances within groups ranged between 0-0.009 (Additional file 7: Table S6). 


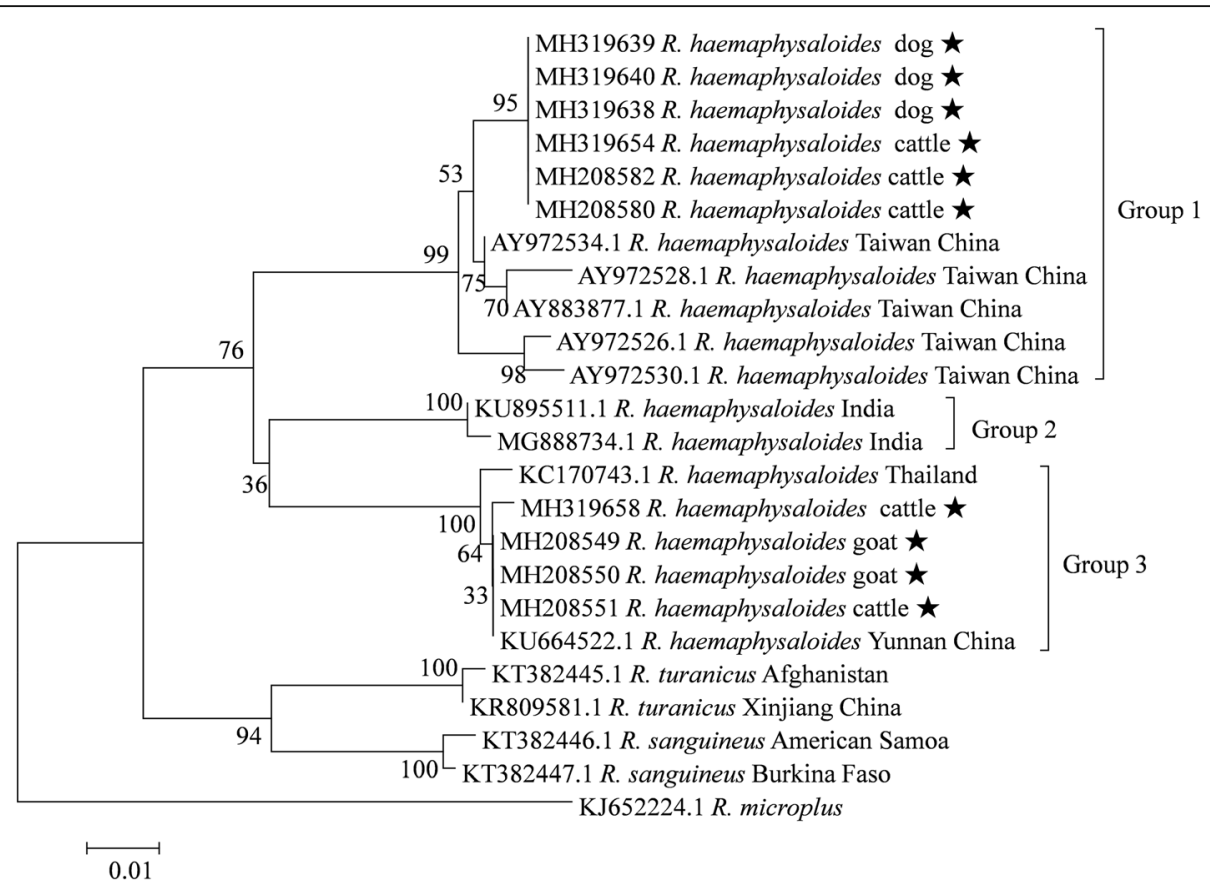

Fig. 3 Phylogenetic tree for R. haemaphysaloides based on the 16S rRNA gene, including sequences obtained in the present study and sequences retrieved from GenBank. The sequences generated in the present study are indicated with a star

Similarly, phylogenetic analysis based on the cox1 gene also revealed that $I$. ovatus ticks clustered into 3 separate groups (Additional file 8: Figure S2). The K2P distances within and between groups ranged between $0.002-0.010$ and $0.142-0.189$, respectively (Additional file 9: Table S7).

\section{Phylogenetic analyses and genetic distances for Haemaphysalis spp.}

According to phylogenetic analysis of the $16 S$ rRNA gene, all specimens of $H$. longicornis from the present study and GenBank formed a single clade (Additional file 10: Figure S3). The pairwise K2P distances of the $16 \mathrm{~S}$ rRNA gene ranged between 0-0.012 (Additional file 11: Table S8). However, $H$. longicornis formed two separate clades based on the cox 1 gene (Fig. 5). Strains from Hebei, Hubei and Gansu of China clustered together, while strains from the present study and those from Australia and Zhejiang, Shanghai, Anhui, Henan of China formed another clade. The pairwise K2P distances between ticks from the two clades ranged between 0.019-0.031, while the distances between ticks from the same clade ranged between 0-0.008 (Additional file 12: Table S9).

For $H$. shimoga ticks, 4 sequences of $16 S$ rRNA gene from the present study were selected for phylogenetic analysis, and 2 genetic groups were recognized: $H$. shimoga collected from India and Thailand clustered together, while all of the specimens from the present study formed a separate group (Fig. 6). Similarly, 2 genetic groups were recognized for $H$. kitaokai: all $H$. kitaokai ticks from this study formed a separate clade, while $H$. kitaokai ticks from Japan clustered together. The intergroup K2P distance ranged between 0.051-0.057 for $H$. shimoga and between 0.054-0.060 for H. kitaokai (Additional file 13: Table S10).

\section{Discussion}

Little is known about the genetic diversity of hard ticks in China, especially at the subspecies level [33]. In the present study, we conducted a survey on hard ticks in a China-Myanmar border county of Yunnan Province and analyzed the molecular characteristics of each tick species based on $16 S$ rRNA and cox1 gene fragments. Several tick species were identified based on morphology, including $R$. microplus, $R$. haemaphysaloides, $I$. ovatus, $H$. longicornis, $H$. shimoga and $H$. kitaokai.

Rhipicephalus microplus was found to be the most predominant tick species in the local area (Table 1). This species is widespread in tropical and subtropical regions and is considered to be the most important tick species infesting livestock in the world. In the present study, the $R$. microplus complex consisted of morphologically similar species difficult to distinguish. 


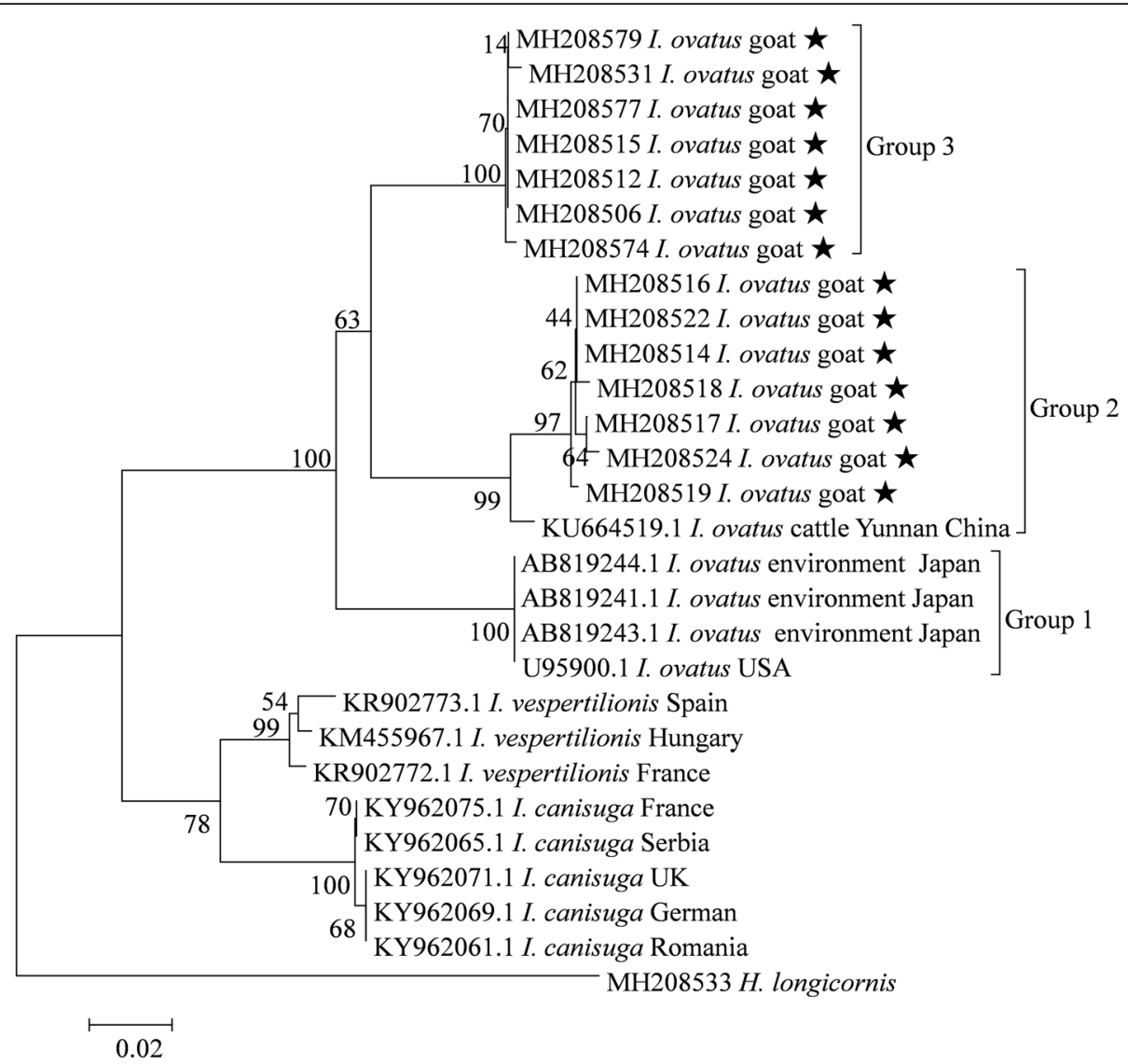

Fig. 4 Phylogenetic tree for I. ovatus based on the 165 rRNA gene, including sequences obtained in the present study and representative sequences from GenBank. The sequences generated in the present study are indicated with a star

At least five taxa have been confirmed by phylogenetic analysis based on DNA barcoding, including $R$. australis, $R$. annulatus and $R$. microplus clades A-C [31, 32]. However, genetic diversity of $R$. microplus in China had not been analyzed. Our study revealed that, except for $R$. australis, all of the other four taxa were detected in China (Additional file 4: Figure S1). Ticks from the present study were clustered into $R$. microplus clade $C$; this clade also contains ticks from Myanmar, Bangladesh and Pakistan [34].

As far as we are aware, the genetic diversity of $R$. haemaphysaloides and I. ovatus had not been previously investigated. Three genetic groups were recognized based on analysis of $16 S$ rRNA gene, and $R$. haemaphysaloides ticks from the present study were found to belong to two of the three groups (Fig. 3). The pairwise genetic distances between ticks from different $R$. haemaphysaloides groups exceeded the recommended boundaries for describing tick species based on the $16 S$ rRNA gene [10]. In addition, the intergroup distances of $R$. haemaphysaloides were higher than the interspecies distances between $R$. sanguineus and $R$. turanicus (Additional file 6: Table S5). Similarly, three groups of I. ovatus were identified based on both genes (Fig. 4 and Additional file 8: Figure S2), and the between-group K2P distances also exceeded the recommended species boundaries for ticks $(0.053$ for the $16 S$ rRNA gene and 0.061 for the cox1 gene) [10, 35] (Additional file 7: Table S6 and Additional file 9: Table S7). Moreover, the genetic distances between ticks from different $I$. ovatus groups were higher than interspecies distances between $I$. vespertilionis and I. canisuga. The results suggest that different groups might represent distinct species or subspecies, or both $R$. haemaphysaloides and I. ovatus are species complexes with high genetic diversity. Unfortunately, morphological comparisons between groups were not performed in the present study. To further determine the taxonomic status of these phylogenetic groups, more studies are warranted to compare their morphological and molecular characteristics.

Few studies have investigated the molecular characteristics of $H$. longicornis. Li et al. [36] analyzed 


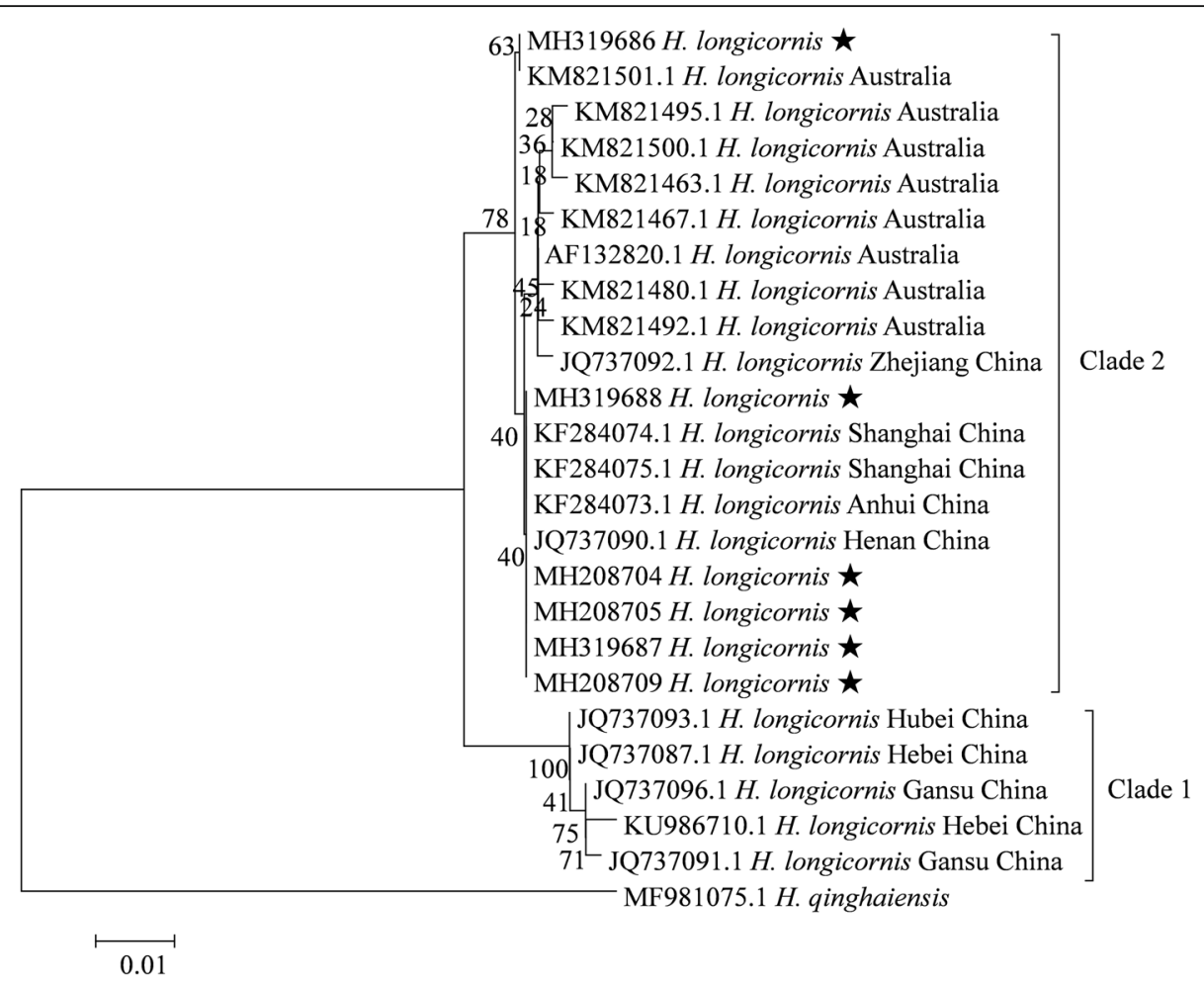

Fig. 5 Phylogenetic tree for $\mathrm{H}$. longicornis based on the cox 1 gene, including sequences obtained in the present study and representative sequences from GenBank. The sequences generated in the present study are indicated with a star

the genetic variation of $H$. longicornis from Hunan, Henan and Shandong provinces using the internal transcribed spacer (ITS) of rDNA data and found that $H$. longicornis formed a monophyletic group. Similarly, all $H$. longicornis ticks formed a single clade based on phylogenetic analysis of the $16 \mathrm{~S}$ $r R N A$ gene in the present study (Additional file 10: Figure S3). However, they were clustered into two separate groups based on the cox 1 gene (Fig. 5) and the intergroup genetic distances were higher than intragroup distances (Additional file 12: Table S9). These results suggest that $\operatorname{cox} 1$ sequences are probably more informative than $16 \mathrm{~S}$ rRNA sequences in revealing the intraspecies phylogenetic relationships [31]. Chen et al. [37] compared the molecular characteristics of parthenogenetic and bisexual $H$. longicornis using the $16 S$ rRNA gene. Their results showed that the genetic distance between the parthenogenetic and bisexual strains was closer than that between subspecies. Similarly, a phylogenetic tree cannot differentiate parthenogenetic strains from bisexual strains. For example, parthenogenetic strains from the present study and Shanghai are clustered together with bisexual strains from other regions (data not shown).
Haemaphysalis shimoga was first described by Trapido \& Hoogstraal in 1964 [29], and H. kitaokai was described by Hoogstraal in 1969 [30]. Little is known about the genetic diversity of these two species. In this study, two genetic groups were recognized for both $H$. shimoga and $H$. kitaokai based on phylogenetic analysis of $16 \mathrm{~S}$ rRNA gene (Fig. 6). Hornok et al. [20] investigated the genetic divergence between ticks of different subspecies of $H$. erinacei. The results suggested that $H$. erinacei taurica and $H$. erinacei turanica might represent different species because the genetic divergence between them exceeded the usual divergence boundary for closely related tick species. In the present study, the intergroup genetic distances of both $H$. shimoga (0.051-0.057) and H. kitaokai (0.054-0.060) exceeded the distances between $H$. erinacei taurica and $H$. erinacei turanica (Additional file 13: Table S10), suggesting that the different groups of $H$. shimoga and H. kitaokai might represent distinct species or subspecies or that both species are species complexes with a high genetic diversity.

\section{Conclusions}

According to the phylogenetic analysis and K2P genetic distances based on $16 \mathrm{~S}$ rRNA and cox1 genes, either 


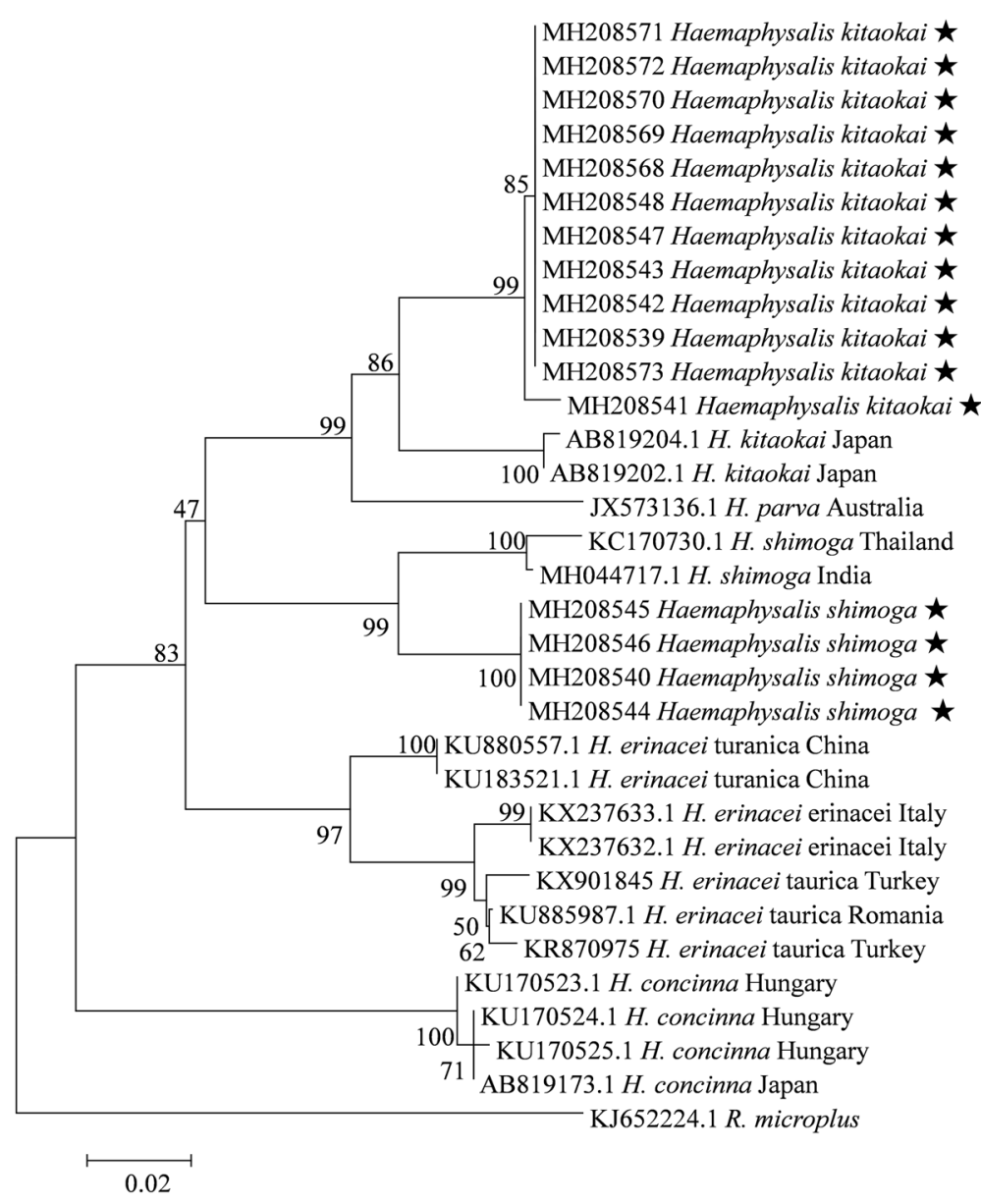

Fig. 6 Phylogenetic tree for H. kitaokai and H. shimoga based on the 16S rRNA gene, including sequences obtained in the present study and representative sequences of closely related tick species from GenBank. The sequences generated in the present study are indicated with a star

new species or subspecies closely related to $R$. haemaphysaloides, I. ovatus, H. shimoga and H. kitaokai might exist in this China-Myanmar border county or these ticks form species complexes with highly divergent mitochondrial lineages. Morphological comparisons are warranted to further confirm the taxonomic status of these tick species.

\section{Additional files}

Additional file 1: Table S1. The accession numbers for the 165 rRNA sequences generated in the present study and the closest species sequences from the GenBank database. (XLSX $20 \mathrm{~kb}$ )

Additional file 2: Table S2. The accession numbers for the cox1 sequences generated in the present study and the closest species sequences from the GenBank database. (XLSX $12 \mathrm{~kb}$ )

Additional file 3: Table S3. The pairwise K2P genetic distances for $R$. microplus and other closely related Rhipicephalus spp. based on the 165 rRNA gene. (XLSX $18 \mathrm{~kb}$ )

Additional file 4: Figure S1. Phylogenetic tree for R. microplus based on the cox 1 gene, including sequences obtained in the present study and representative sequences of the known subspecies taxa from GenBank. Sequences obtained in this study are designated by an asterisk. (TIF $1139 \mathrm{~kb})$

Additional file 5: Table S4. The pairwise K2P genetic distances for $R$. microplus and other closely related Rhipicephalus spp. based on the cox1 gene. (XLSX $17 \mathrm{~kb})$

Additional file 6: Table S5. The pairwise K2P genetic distances for $R$. haemaphysaloides and other closely related Rhipicephalus spp. based on the 165 rRNA gene. (XLSX $22 \mathrm{~kb}$ )

Additional file 7: Table S6. The pairwise K2P genetic distances for $I$. ovatus and other closely related lxodes spp. based on the 165 rRNA gene. (XLSX $19 \mathrm{~kb}$ )

Additional file 8: Figure S2. Phylogenetic tree for I. ovatus based on the cox1 gene, including sequences obtained in the present study and representative sequences from GenBank. Sequences obtained in this study are designated by an asterisk. (TIF $712 \mathrm{~kb}$ )

Additional file 9: Table S7. The pairwise K2P genetic distances for $I$. ovatus and other closely related lxodes spp. based on the cox1 gene. (XLSX $13 \mathrm{~kb}$ )

Additional file 10: Figure S3. Phylogenetic tree for $\mathrm{H}$. longicornis based on the 165 rRNA gene, including sequences obtained in the present study and representative sequences from GenBank. Sequences obtained in this study are designated by an asterisk. (TIF 1672 kb) 
Additional file 11: Table S8. The pairwise K2P genetic distances for $H$ longicornis based on the 165 rRNA gene. (XLSX $45 \mathrm{~kb}$ )

Additional file 12: Table S9. The pairwise K2P genetic distances for $H$. longicornis based on the cox1 gene. (XLSX $13 \mathrm{~kb}$ )

Additional file 13: Table S10. The pairwise K2P distances of $H$. kitaokai, H. shimoga and other closely related species based on the 16S rRNA gene. (XLSX $14 \mathrm{~kb})$

\section{Abbreviations}

BLAST: Basic Local Alignment Search Tool; cox1: cytochrome c oxidase subunit 1; K2P: Kimura's 2-parameter; NJ: neighbor-joining

\section{Acknowledgments}

We thank the village doctors from each location for their help with tick collection.

\section{Funding}

The research has been partially supported by the National Key Research and Development Program of China (nos. 2016YFC1200500 and 2016YFC1202000), the Special Fund for Health Research in the Public Interest China (no. 201202019), Health Shandong Collaborative Innovation Program for Major Social Risk Prediction and Management (no. XT1404001), and the Scientific Research Foundation for Doctors of Weifang Medical College (no. 2017BSQD52)

\section{Availability of data and materials}

The data supporting the conclusions of this article are included within the article and its additional files. The sequences generated in the present study were submitted to GenBank under the accession numbers MH208505MH208600; MH208680-MH208709; and MH319485-MH319688.

\section{Authors' contributions}

LHL designed the investigation, performed the experiments and drafted the manuscript. YZ designed the investigation and revised the manuscript. JZW, XSL, SQY, DZ and JBX conducted the investigation. SGL conceived the study and conducted the investigation. All authors read and approved the final manuscript.

\section{Ethics approval and consent to participate}

No specific permits were required for this study. The study did not involve endangered or protected species. Therefore, the local ethics committee deemed that approval was unnecessary.

\section{Consent for publication}

Not applicable.

\section{Competing interests}

The authors declare that they have no competing interests.

\section{Publisher's Note}

Springer Nature remains neutral with regard to jurisdictional claims in published maps and institutional affiliations.

\footnotetext{
Author details

${ }^{1}$ Health Shandong Collaborative Innovation Center for Major Social Risk Prediction and Management, School of Public Health and Management, Weifang Medical University, Weifang 261053, People's Republic of China. ${ }^{2}$ National Institute of Parasitic Diseases, Chinese Center for Disease Control and Prevention, Shanghai 200025, People's Republic of China. ${ }^{3}$ Tengchong Center for Disease Control and Prevention, Tengchong 679100, People's Republic of China.
}

Received: 11 May 2018 Accepted: 1 August 2018 Published online: 14 August 2018

\section{References}

1. Yu Z, Wang H, Wang T, Sun W, Yang X, Liu J. Tick-borne pathogens and the vector potential of ticks in China. Parasit Vectors. 2015;8:24.
2. Qin XC, Shi M, Tian JH, Lin XD, Gao DY, He JR, et al. A tick-borne segmented RNA virus contains genome segments derived from unsegmented viral ancestors. Proc Natl Acad Sci USA. 2014;111:6744-9.

3. Mansfield $K L$, Jizhou L, Phipps LP, Johnson N. Emerging tick-borne viruses in the twenty-first century. Front Cell Infect Microbiol. 2017;7:298.

4. Jongejan F, Uilenberg G. The global importance of ticks. Parasitology. 2004; 129(Suppl.):S3-S14.

5. Chen Z, Yang X, Bu F, Yang X, Yang X, Liu J. Ticks (Acari: Ixodoidea: Argasidae, Ixodidae) of China. Exp Appl Acarol. 2010;51:393-404.

6. Liu JF, Bi ZQ, Liu GH, Ren J, Wang H, Zhao Z, et al. Fever with thrombocytopenia associated with a novel bunyavirus in China. New Engl J Med. 2011;364:1523-32.

7. Fang LQ, Liu K, Li XL, Liang S, Yang Y, Yao HW, et al. Emerging tick-borne infections in mainland China: an increasing public health threat. Lancet Infect Dis. 2015:15:1467-79.

8. Zhuang L, Du J, Cui XM, Li H, Tang F, Zhang PH, et al. Identification of tickborne pathogen diversity by metagenomic analysis in Haemaphysalis longicornis from Xinyang, China. Infect Dis Poverty. 2018;7:45

9. Man SQ, Qiao K, Cui J, Feng M, Fu YF, Cheng XJ. A case of human infection with a novel Babesia species in China. Infect Dis Poverty. 2016;5:28.

10. Lv J, Wu S, Zhang Y, Chen Y, Feng C, Yuan X, et al. Assessment of four DNA fragments (COI, 16S rDNA, ITS2, 12S rDNA) for species identification of the xodida (Acari: Ixodida). Parasit Vectors. 2014;7:93.

11. Zhou X, Li SG, Chen SB, Wang JZ, Xu B, Zhou HJ, et al. Co-infections with Babesia microti and Plasmodium parasites along the China-Myanmar border. Infect Dis Poverty. 2013;2:24.

12. Deng G, Jiang ZJ. Economic Insect Fauna of China, volume 39 (Acari: Ixodidae). Beijing: Science Press; 1988. p. 1-358.

13. Chen Z, Liu Q, Liu JQ, Xu BL, Lv S, Xia S, Zhou XN. Tick-borne pathogens and associated co-infections in ticks collected from domestic animals in central China. Parasit Vectors. 2014;7:237.

14. Fang Y, Shi WQ, Zhang Y. Molecular phylogeny of Anopheles hyrcanus group (Diptera: Culicidae) based on mtDNA COI. Infect Dis Poverty. 2017;6:61

15. Zemtsova GE, Apanaskevich DA, Reeves WK, Hahn M, Snellgrove A, Levin ML. Phylogeography of Rhipicephalus sanguineus sensu lato and its relationships with climatic factors. Exp Appl Acarol. 2016;69:191-203.

16. Takano A, Fujita H, Kadosaka T, Takahashi M, Yamauchi T, Ishiguro F, et al. Construction of a DNA database for ticks collected in Japan: application of molecular identification based on the mitochondrial 16S rDNA gene. Med Entomol Zool. 2014;65:13-21.

17. Hornok S, Sándor AD, Beck R, Farkas R, Beati L, Kontschán J, et al. Contributions to the phylogeny of Ixodes (Pholeoixodes) canisuga, I. (Ph.) kaiseri, I. (Ph.) hexagonus and a simple pictorial key for the identification of their females. Parasit Vectors. 2017;10:545

18. Hornok S, Kontschán J, Estrada-Peña A, de Mera IGF, Tomanović S, de la Fuente J. Contributions to the morphology and phylogeny of the newly discovered bat tick species, Ixodes ariadnae in comparison with I. vespertilionis and I. simplex. Parasit Vectors. 2015:8:47.

19. Ernieenor F, Ernna G, Mariana A. Phenotypic and genotypic identification of hard ticks of the genus Haemaphysalis (Acari: Ixodidae) in Peninsular Malaysia. Exp Appl Acarol. 2017;71:387-400.

20. Hornok S, Wang Y, Otranto D, Keskin A, Lia RP, Kontschán J, et al. Phylogenetic analysis of Haemaphysalis erinacei Pavesi, 1884 (Acari: Ixodidae) from China, Turkey, Italy and Romania. Parasit Vectors. 2016;9:643.

21. Burger TD, Shao R, Barker SC. Phylogenetic analysis of the mitochondria genomes and nuclear rRNA genes of ticks reveals a deep phylogenetic structure within the genus Haemaphysalis and further elucidates the polyphyly of the genus Amblyomma with respect to Amblyomma sphenodonti and Amblyomma elaphense. Ticks Tick Borne Dis. 2013;4:265-74.

22. Malaisri P, Hirunkanokpun S, Baimai V, Trinachartvanit W, Ahantarig A. Detection of Rickettsia and Anaplasma from hard ticks in Thailand. J Vector Ecol. 2015:40:262-8.

23. Chen X, Xu S, Yu Z, Guo L, Yang S, Liu L, et al. Multiple lines of evidence on the genetic relatedness of the parthenogenetic and bisexual Haemaphysalis longicornis (Acari: Ixodidae). Infect Genet Evol. 2014;21:308-14

24. Kumar S, Tamura K, Nei M. MEGA: Molecular Evolutionary Genetics Analysis software for microcomputers. Comput Appl Biosci. 1994;10:189-91.

25. Brites-Neto J, Duarte KM, Martins TF. Tick-borne infections in human and animal population worldwide. Vet World. 2015;8:301-15. 
26. Zheng W, Chen H, Liu X, Guo X, Fu R. Severe tick infestation in a hare and potential risk for transmitting pathogens to humans. Korean J Parasitol. 2011;49:419-22.

27. Chao LL, Liu LL, Ho TY, Shih CM. First detection and molecular identification of Borrelia garinii spirochete from Ixodes ovatus tick ectoparasitized on stray cat in Taiwan. PLoS One. 2014;9:e110599.

28. Hoogstraal H, Clifford CM, Saito Y, Keirans JE. Ixodes (Partipalpiger) ovatus Neumann, subgen. nov: identity, hosts, ecology, and distribution (Ixodoidea: Ixodidae). J Med Entomol. 1973;10:157-64.

29. Trapido H, Hoogstraal H. Haemaphysalis cornigera shimoga subsp. n. from southern India (Ixodoidea, Ixodidae). J Parasitol. 1964;50:303-10.

30. Hoogstraal H. Haemaphysalis (Alloceraea) kitaokai sp. n. of Japan, and keys to species in the structurally primitive subgenus Alloceraea Schulze of Eurasia (Ixodoidea, Ixodidae). J Parasitol. 1969;55:211-21.

31. Low VL, Tay ST, Kho KL, Koh FX, Tan TK, Lim YA, et al. Molecular characterisation of the tick Rhipicephalus microplus in Malaysia: new insights into the cryptic diversity and distinct genetic assemblages throughout the world. Parasit Vectors. 2015;8:341.

32. Burger TD, Shao R, Barker SC. Phylogenetic analysis of mitochondrial genome sequences indicates that the cattle tick, Rhipicephalus (Boophilus) microplus, contains a cryptic species. Mol Phylogenet Evol. 2014;76:241-53.

33. Lu X, Lin XD, Wang JB, Qin XC, Tian JH, Guo WP, et al. Molecular survey of hard ticks in endemic areas of tick-borne diseases in China. Ticks Tick Borne Dis. 2013;4:288-96.

34. Roy BC, Estrada-Pena A, Krucken J, Rehman A, Nijhof AM. Morphological and phylogenetic analyses of Rhipicephalus microplus ticks from Bangladesh, Pakistan and Myanmar. Ticks Tick Borne Dis. 2018; https://doi.org/10.1016/j.ttbdis.2018.03.035.

35. Apanaskevich DA, Horak IG, Matthee CA, Matthee S. A new species of Ixodes (Acari: Ixodidae) from South African mammals. J Parasitol. 2011;97:389-98.

36. Li ZB, Liu GH, Cheng TY. Molecular characterization of hard tick Haemaphysalis longicornis from China by sequences of the internal transcribed spacers of ribosomal DNA. Exp Appl Acarol. 2018;74:171-6.

37. Chen Z, Yang X, Bu F, Yang X, Liu J. Morphological, biological and molecular characteristics of bisexual and parthenogenetic Haemaphysalis longicornis. Vet Parasitol. 2012;189:344-52.

Ready to submit your research? Choose BMC and benefit from:

- fast, convenient online submission

- thorough peer review by experienced researchers in your field

- rapid publication on acceptance

- support for research data, including large and complex data types

- gold Open Access which fosters wider collaboration and increased citations

- maximum visibility for your research: over $100 \mathrm{M}$ website views per year

At $\mathrm{BMC}$, research is always in progress.

Learn more biomedcentral.com/submissions 